\title{
Effect of friction speed on wear intensity in abrasive soil mass
}

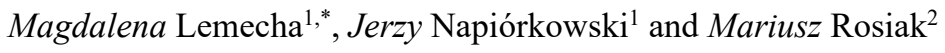 \\ ${ }^{1}$ University of Warmia nad Mazury in Olsztyn, Faculty of Technical Sciences Chair of Building, \\ Operation of Vechicles and Machines, M. Oczapowskiego 11, 10-079 Olsztyn, Poland \\ ${ }^{2}$ Opole University of Technology, Faculty of Mechanical Engineering, Department of Manufacturing \\ Engineering and Production Automation, S.Mikołajczyka 5, 45-271 Opole, Poland
}

\begin{abstract}
One of the major problems when machining soil mass is wear of working tools. The process consists of many factors related to, amongst other things, the work item, the properties of the soil and the working process parameters. During surgeries working big impact on the consumption process parameters are working, such as the type of cutting, machining depth and speed of its implementation. So far, there is no work that would have recognized the issue of the impact of cutting speed on the course wear. Processes which occur in the surface layer material are associated with changes in its mechanical properties, physical and chemical properties. The paper analyses the process of wear steel 38GSA and the same steel weld electrode EstelMn60. Studies were conducted in laboratory conditions by the "spinning bowl" method. During the tests carried out etering of samples depending on the type of road friction and the treated pulp. The results found that with increasing speed decreases wear intensity weight steel 38GSA. And for the deposit EstelMn60 there was no clear effect of speed on wear intensity. It is also noted that a significant impact on the amount of abrasion is the percentage of alloying elements..
\end{abstract}

\section{Introduction}

Wear is defined as a process in which the top layer is changed by gradually removing material from the surface of a solid which is characterised by a permanent deformation or mass loss [1]. Abrasive wear is the most common type of machine working part wearing [2,3]. It usually consists in moving a hardened or loose abrasive material against the surface of a working part, which is in direct contact with the material being processed. It is difficult to control since it is a natural process.

Considering the high intensity reported by many authors [4-6], it is a serious scientific and technical issue. In most papers, the wear of machine working parts in soil mass has been regarded as wear caused by single-cycle separation of material from the surface. Properties of whole soil as abrasive mass are disregarded. Such system-creating factors as the disorderly arrangement of a large number of abrasive grains on the active cutting surface, non-ductility of the abrasion surface, free movement of abrasive grains depending on the condition of soil

\footnotetext{
*Corresponding author: magdalena.lemecha@uwm.edu.pl
} 
mass and the action on the working part of organic mass whose properties depend on the natural surroundings are not taken into account. It has been found that wear of construction materials in soil mass is affected by physicochemical properties of soil and of the construction material, as well as by the parameters of the work process [7-9]. The most important of them include mechanics of cooperation of the working part and soil mass, cutting depth and working speed. Especially the latter of these parameters has not been addressed to a sufficient extent in the literature.

The effect of the cutting speed on wear is usually considered equivalent to friction work [10]. Friction work alone can bring about various effects of wear. The heat which is associated with the friction speed in the surface layer of material brings about changes in the chemical, mechanical and physical properties. Hardness decreases with increasing temperature, which brings about structural changes in the surface layer and affects strains. Friction work can generate various effects, which are caused by such components as: friction speed and friction coefficient, as well as normal pressure during the cutting process. It has been found for steel-steel friction that the wear intensity increases up to the speed of $0.5 \mathrm{~m}^{-\mathrm{s}}$, and then decreases rapidly to $2 \mathrm{~m}^{-\mathrm{s}}$ [11].

Therefore, the relationship which defines the friction force as a function of the speed of the bodies in contact against each other is important for bodies in movement with friction. It is usually assumed in engineering calculations that the relationship is constant, although in fact it is not. It was shown in a study conducted by Stribeck that friction force decreases with increasing relative speed of bodies (within a range of small sliding speed). It is of particular importance in initiating stick-slip movements [12].

Considering the fact that no studies have been found regarding the relationship between the cutting speed and friction force, or wear intensity in soil mass, the objective of this study was to evaluate the effect of cutting speed on wear of various construction materials in diverse abrasive soil masses

\section{Study methodology}

The study was conducted in laboratory conditions on a "centrifugal bowl" machine. $30 \mathrm{x}$ $20 \times 10 \mathrm{~mm}$ samples were made of 38GSA steel and of the same steel pad-welded with an EstelMn60 electrode. The structure of the surface layers was assessed metalographically under an optical microscope - Neophot 52 - coupled with a Visitron Systems digital camera and by electron scanning microscopy. A microanalysis of the chemical composition was conducted with a JEOL JSM-5800 LV scanning microscope coupled with an Oxford LINK ISIS-300 X-Ray microanalyser.

38GSA is a steel of martensitic structure, containing perlite and bainite (Fig. 1). EstelMn60 welded pad is a material of a plate structure, containing residual austenite and primary carbides $\left.\left[\alpha+(\mathrm{Fe}, \mathrm{Cr})_{7} \mathrm{C}_{3}\right)\right]$ with numerous carbides $\left(\mathrm{Fe}, \mathrm{Cr}_{3}\right) \mathrm{C}$ (Fig. 2$)$. The chemical composition of the steel and the welded pad is shown in Table 1. Hardness was measured with a Vickers hardness tester, type HM010u, in accordance with PN-EN ISO 6507-1:1999. The indenting tool was loaded with a weight of $98 \mathrm{~N}$, loading time - $10 \mathrm{~s}$. The steel hardness was 436 HV10, and that of the welded pad -697 HV10. Sand and clay were used as the abrasive mass. Granulation was classified in accordance with PN- EN ISO 14668-2(2004).

The abrasive soil mass taken for the study and its parameters are shown in Table 2.

Weight wear was determined from the formula:

$$
Z_{w}=m_{w}-m_{i}
$$

where: $\mathrm{m}_{\mathrm{w}}$ - starting weight of a sample $[\mathrm{g}], \mathrm{m}_{\mathrm{i}}$ - weight of a sample after it completed the friction distance $[\mathrm{g}]$. 


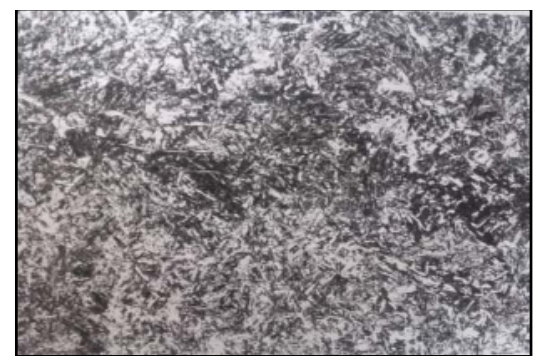

Fig. 1. Steel 38GSA. Martensite and bainite with perlite fine grained. Magnification $500 x$.

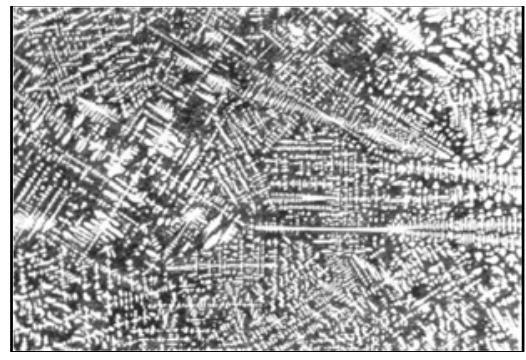

Fig. 2. EStelMn60 surfacing weld: The distribution of primary carbides in a matrix of eutectic structure of the leaf + retained austenite. Magnification 200x.

Table 1. Chemical composition of the materials tested.

\begin{tabular}{|c|c|c|}
\hline $\begin{array}{c}\text { Element } \\
\text { (symbol) }\end{array}$ & $\begin{array}{c}\text { 38GSA } \\
\text { etceteras [\%] }\end{array}$ & $\begin{array}{c}\text { EStelMn 60 } \\
\text { etceteras [\%] }\end{array}$ \\
\hline $\mathrm{C}$ & 0.35 & 2.99 \\
\hline $\mathrm{Mn}$ & 1.07 & 6.28 \\
\hline $\mathrm{Si}$ & 1.17 & 1.68 \\
\hline $\mathrm{P}$ & 0.03 & 0.05 \\
\hline $\mathrm{S}$ & 0.02 & 0.14 \\
\hline $\mathrm{Cr}$ & 0.18 & 31.6 \\
\hline $\mathrm{Ni}$ & 0.09 & 0.30 \\
\hline $\mathrm{Cu}$ & 0.16 & 0.09 \\
\hline $\mathrm{Al}$ & 0.022 & 0.04 \\
\hline $\mathrm{Mo}$ & - & 0.04 \\
\hline $\mathrm{V}$ & - & 0.07 \\
\hline $\mathrm{Ti}$ & - & 0.10 \\
\hline $\mathrm{B}$ & - & 0.002 \\
\hline
\end{tabular}

Table 2. Characteristics of soil particle size.

\begin{tabular}{|c|c|c|c|c|}
\hline \multirow{2}{*}{ Soil } & \multicolumn{4}{|c|}{ \% the fraction with a diameter in mm } \\
\cline { 2 - 5 } & gravel $>\mathbf{1 . 0}$ & sand 0.1-1.0 & dust 0.02-0.1 & loam <0.02 \\
\hline Sand & 0.8 & 71.6 & 21.0 & 7.4 \\
\hline Clay & 0.4 & 28.4 & 25.6 & 46.0 \\
\hline
\end{tabular}

Three friction speeds were taken for the experiment, which usually occur in cutting soil mass, i.e. $0.56,1.39$ and $2.00 \mathrm{~m}^{-s}$. Unit pressure $57 \mathrm{kPa}$. Total friction distance was $2,000 \mathrm{~m}$. The samples were weighed every $2,000 \mathrm{~m}$ and the soil mass was replaced. The soil moisture content was determined by measuring the weight of the solid phase dried at $105^{\circ} \mathrm{C}$, which ranged from $10 \%$ to $12 \%$. Before the test, the samples were washed in an ultrasonic washer with a potassium hydroxide-based washing agent. 


\section{Test results and analysis}

Figures 3-4 show the wear of the construction material at various speeds.

a)

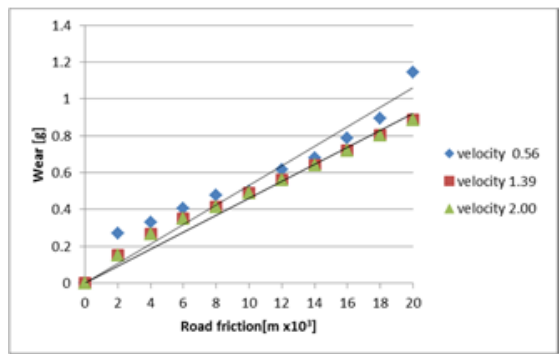

b)

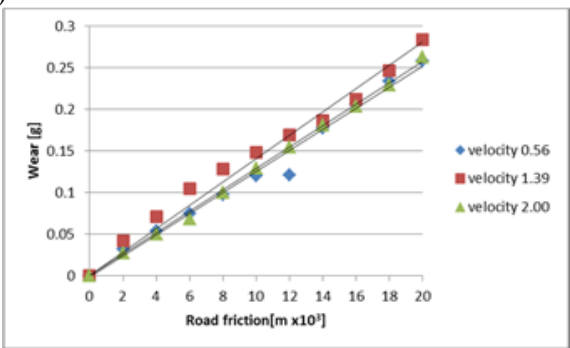

Fig. 3. The course of wear in the sand: a) 38 GSA steel, b) EStelMn60 welded.

a)

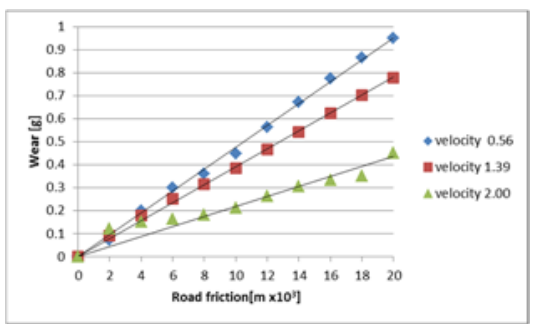

b)

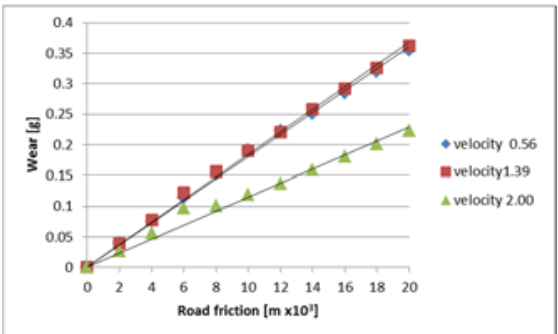

Fig. 4. The course of wear in clay: a) 38 GSA steel, b) EStelMn60 welded.

The results have shown no significant effect of the cutting speed on the wearing intensity of 38GSA steel in clayey soil mass. The most intensive wear was recorded at the speed of $\mathrm{V}=0.56 \mathrm{~ms}^{-1}$, and the least intensive was at the speed of $\mathrm{V}=2.00 \mathrm{~ms}^{-1}$. The wearing analysis of the EStelMn60 welded pad did not show any significant differences in the wear intensity (see Fig. 5).

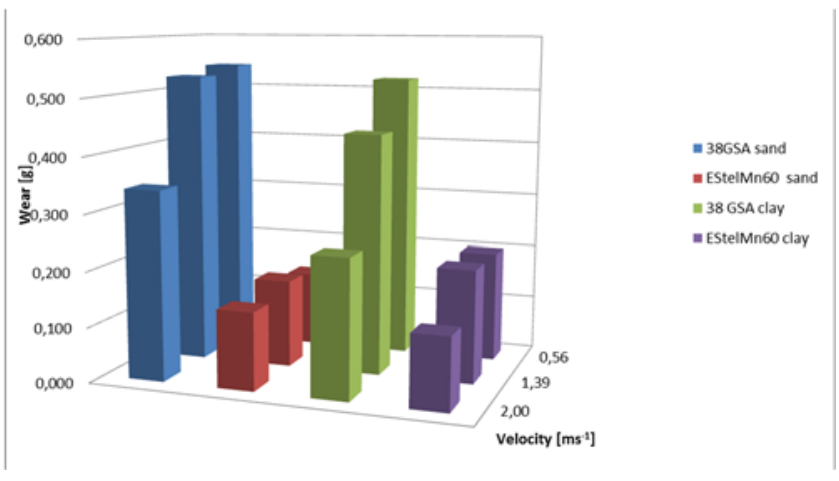

Fig. 5. Compilation of total consumption of materials in studied soils.

The results of the statistical analysis conducted to identify significant wearing intensity differences between the materials under study in sand and clay showed that cutting speed has 
a significant effect on the wearing intensity, both for 38GSA steel and for the EStelMn60 welded pad (Table 3, 4).

Table 3. Duncan's statistics differ in the intensity of wear depending on the cutting speed.

\begin{tabular}{|c|c|c|c|c|}
\hline \multirow{2}{*}{ Soil } & \multicolumn{3}{|c|}{ Duncan test } & $\mathbf{1}$ \\
\cline { 2 - 5 } & Materials & Average consumption & $* * * *$ & \\
\hline Sand & EStelMn60 & 0.1455 & & $* * * *$ \\
\hline Sand & $38 \mathrm{GSA}$ & 0.4888 & & $\mathbf{2}$ \\
\hline
\end{tabular}

**** - it illustrates the statistically significant difference $\mathrm{p}<0.05$ between different grades of steel in a particular soil species.

Tabele 4. Duncan's statistics differ in the intensity of wear depending on the cutting speed.

\begin{tabular}{|c|c|c|c|c|}
\hline \multirow{2}{*}{ Soil } & \multicolumn{3}{|c|}{ Duncan test } & $\mathbf{1}$ \\
\cline { 2 - 5 } & Materials & Average consumption & $* * * *$ & \\
\hline Clay & EStelMn60 & 0.1782 & & $* * * *$ \\
\hline Clay & $38 G S A$ & 0.4002 & & 2 \\
\hline
\end{tabular}

**** - it illustrates the statistically significant difference $\mathrm{p}<0.05$ between different grades of steel in a particular soil species.

This is caused by the smallest percentage of silt and clay fractions in this type of abrasive mass. These finest fractions of abrasive mass, in combination with moisture, are responsible for removing the matrix from the surface of the sintered carbide samples. A higher percentage of fine fractions in abrasive mass increases the wearing intensity of sintered carbides.

\section{Summary}

This study showed that the cutting speed affects the wear of working parts, depending on the material properties and type of soil. The process is affected by the soil contact with the surface layer of a construction material. The contact time of soil grains in sandy soil is much shorter than in clayey soil. Abrasive grains in sandy soil have a considerable number of degrees of freedom. The contact with a construction material is discrete regardless of the friction speed. For clayey soils, a high percentage of silty and clayey fractions (71.6\%) makes a soil under load acquire properties of a continuous medium. Soil is under plastic deformations at low speeds and adhesion on the friction surface takes place. With increasing speed, the elastic strain of soil mass begins to dominate, which changes the wearing intensity.

The same relationships were found for both construction materials, despite different surface layer characteristics. The wear of the EStelMn60 welded pad was equal to half of that of 38 GSA steel, regardless of the speed or the type of soil mass.

Apart from the cutting speed, the wear process is affected by the susceptibility of the surface layer of steel and the welded pad. It is noteworthy that no significant effect of temperature was observed as the processed soil consistently took away the energy generated in the process.

\section{References}

1. A. Zmitrowicz, JTAM, 44, 219-253 (2006)

2. R.W. Maruda, G.M. Krolczyk, E. Feldshtein, P. Nieslony, B. Tyliszczak, F. Pusavec, Wear, 372-373, 54-67 (2017) 
3. R.W. Maruda, G.M. Krolczyk, P. Nieslony, S. Wojciechowski, M. Michalski, S. Legutko, J Manuf Process, 24, 107-115 (2016)

4. A. Gierek, Tribological consumption. Gliwice: The Silesian Technical University (2005)

5. R. Maruda, E. Feldshtein, S. Legutko, G.M. Krolczyk, J Frict Wear+, 36, 548 - 553 (2015)

6. J. Napiórkowski, (ed.), P. Szczyglak, P. Drożyner, K. Ligier, P. Mikolajczak, A. Rychlik, $\mathrm{K}$. Kolakowski. Research and modeling of abrasive and fatigue wear processes. (in Polish) (Olsztyn: University of Warmia and Mazury, 2014)

7. M. Nalbant, A.T. Palali, TURJAF, 35, 215-223 (2011)

8. J. Napórkowski, Spatial impact of soil on working elements of agricultural tools (habilitation thesis). Agricultural Engineering, 12, 72 (2005)

9. P. Kostencki, B. Letkowska, R. Nowowiejski, (in Polish), Tribologia 44 (3), 49-79 (2013)

10. Y. Bayhan, Tribology International 39 (6), 570-574 (2006)

11. U. König, H. Grewe Investigations on the production and properties of hard material layers produced by reactive vapor deposition. 197 -250 Tribology 1, (Berlin: Springer 1981)

12. M. Wiercigroch, JSV, 175(5), 700-704 (1994) 